Euller Duarte de Carvalho'

Ana LúCia Riberro Valadares ${ }^{2}$

lúcia Helena da Costa-Paiva ${ }^{3}$

AdRIANa ORCEsI Pedro ${ }^{4}$

SIRLEI SIANI MORAIS ${ }^{5}$

Aarão Mendes Pinto-Neto ${ }^{6}$

Artigo original

Palavras-chave

Estilo de vida sedentário

Envelhecimento

Saúde da mulher

Qualidade de vida

Exercício

Keywords

Sedentary lifestyle

Aging

Women's health

Quality of life

Exercise

Aarão Mendes Pinto-Neto

Departamento de Tocoginecologia da Faculdade de Ciências Médicas da Universidade Estadual de Campinas (UNICAMP)

Rua Alexandre Fleming, 101 - Cidade Universitária "Zeferino Vaz"

Barão Geraldo

CEP 13083-881 - Campinas (SP), Brasi

Caixa Postal 6081

E-mail: aarao@unicamp.br

Recebido

$22 / 7 / 10$

Aceito com modificaç̃es

$25 / 8 / 10$

\section{Atividade física e qualidade de vida em mulheres com 60 anos ou mais: fatores associados}

\author{
Physical activity and quality of life in women aged 60 or older: \\ associated factors
}

\section{Resumo}

OBJETIVO: avaliar o nível de atividade física, a qualidade de vida e os fatores associados em mulheres com 60 anos ou mais. MÉTODOS: estudo de corte transversal que incluiu 271 mulheres frequentadoras de um centro de lazer e de mulheres atendidas no Ambulatório de Menopausa em Campinas (SP). As mulheres foram convidadas a participar da pesquisa, que foi realizada com o uso de entrevistas. Os instrumentos utilizados foram o International Physical Activity Questionnaire (IPAQ), versão 8, modificado para a população idosa para avaliar o nível de atividade física, e o Questionário de Qualidade de Vida da Organização Mundial da Saúde, específico para este grupo (WHOQOLOLD), para avaliar o escore de qualidade de vida. Os resultados do IPAQ foram avaliados por meio de tercis, e a associação entre resultados do WHOQOL-OLD e IPAQ e características das mulheres pelos testes t de Student/ Mann-Whitney e de análises múltiplas. RESULTADOS: a média etária das mulheres foi de 67,4 5,3 anos. Destas, $33 \%$ foram classificadas como pouco ativas. A análise de cada domínio da atividade física mostrou que 60,8\% do

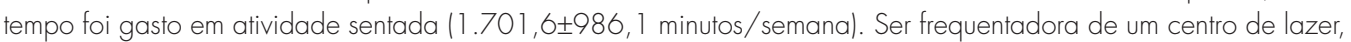
ter maior idade, sem companheiro, maior escolaridade e boa autopercepção do estado de saúde, sem antecedentes de doenças e maior renda foram características que se associaram significativamente à prática de exercícios físicos de intensidade moderada/vigorosa. A análise múltipla evidenciou que frequentar um centro de lazer em Campinas (SP) e ter 70 anos ou mais aumentaram a chance de praticar exercícios físicos de intensidade moderada ou vigorosa,

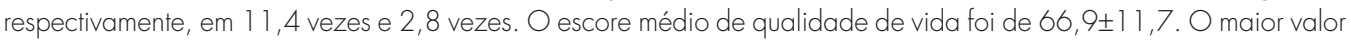
foi observado no domínio referente às habilidades sensoriais $(72,0 \pm 18,8)$, e o menor no que se refere à autonomia $(60,3 \pm 16,2)$. A regressão linear mostrou que a boa autopercepção da saúde aumentou o escore de qualidade de vida em 7,3 pontos; o uso de maior número de medicamentos diminuiu em 4,4 pontos; e a prática de exercícios físicos moderados ou vigorosos aumentou em 4,8 pontos o referido escore. CONCLUSÕES: as mulheres despendem muito tempo na posição sentada. Evidenciou-se a importância da prática de exercícios físicos de intensidade moderada/ vigorosa contribuindo para a obtenção de uma boa qualidade de vida.

\section{Abstract}

PURPOSE: to evaluate the level of physical activity, quality of life and associated factors in women aged 60 or older. METHODS: a cross-sectional study was conducted on 271 women who go to a Leisure Center and women attended at a menopause ambulatory in Campinas (SP). The women were invited to take part in the research, carried out through interviews. The instruments used were the version 8 of the International Physical Activity Questionnaire (IPAQ) modified for the elderly population in order to evaluate their physical activity, and the World Health Organization Questionnaire of Quality of Life specific for this group (WHOQOL-OLD) to evaluate their quality of life. IPAQ results were assessed using tertiles. The association between the WHOQOL-OLD and the IPAQ results and subject characteristics was assessed by the Student's $t$ test, Mann-Whitney test and multiple analyses. RESULTS: the average age of women was $67.4 \pm 5.3$ years. Among these women, $33 \%$ were classified as being less active. Analysis of each physical activity domain

Departamento de Tocoginecologia da Faculdade de Ciências Médicas da Universidade Estadual de Campinas - UNICAMP Campinas (SP), Brasil.

'Pós-graduando (Mestrado) pelo Programa de Pós-graduação do Departamento de Tocoginecología da Faculdade de Ciências Médicas da Universidade Estadual de Campinas - UNICAMP - Campinas (SP), Brasil.

2 Pós-Doutoranda do Departamento de Tocoginecologia da Faculdade de Ciências Médicas da Universidade Estadual de Campinas - UNICAMP - Campinas (SP), Brasil.

${ }_{3}^{3}$ Professora Livre-Docente do Departamento de Tocoginecologia da Faculdade de Ciências Médicas da Universidade Estadual de Campinas - UNICAMP - Campinas (SP), Brasil.

4 Professora Doutora Departamento de Tocoginecologia da Faculdade de Ciências Médicas da Universidade Estadual de Campinas - UNICAMP - Campinas (SP), Brasil.

${ }^{5}$ Estatística do Departamento de Tocoginecologia da Faculdade de Ciências Médicas da Universidade Estadual de Campinas UNICAMP - Campinas (SP), Brasil

- Professor Titular do Departamento de Tocoginecologia da Faculdade de Ciências Médicas da Universidade Estadual de Campinas - UNICAMP - Campinas (SP), Brasil. 


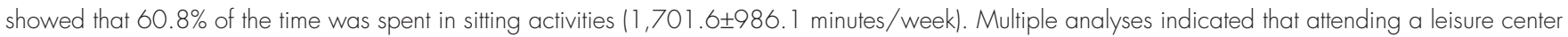
in Campinas (SP) and being 70 years old or older increased the chances of engaging in moderate-intensity or vigorous-intensity physical activity by 11.4

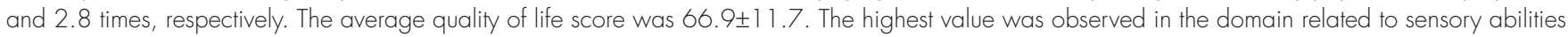
(72.0 \pm 18.8 ) and the lowest value was related to autonomy (60.3 \pm 16.2$)$. Linear regression showed that a good self-perception of health increased the quality of life score by 7.3 points, the use of a bigger amount of medication decreased it by 4.4 points and the performance of moderate or vigorous physical exercise increased the score by 4.8 points. CONCLUSION: women spend prolonged periods of time in sitting activities. The importance of engaging in moderate/vigorous-intensity physical activity is evident for obtaining a good quality of life.

\section{Introdução}

O envelhecimento refere-se a uma função fisiológica e de comportamento social. É um processo de regressão, comum a todos os seres vivos, influenciado por fatores genéticos, estilo de vida e características sócio e psicoemocionais ${ }^{1}$. Pode-se dizer que é dinâmico e progressivo, com alterações morfológicas, funcionais e bioquímicas, reduzindo a capacidade de adaptação homeostática às situações de sobrecarga funcional, modificando gradativamente o organismo e deixando-o mais frágil às agressões intrínsecas e extrínsecas ${ }^{2}$. Associa-se ao aumento de massa de gordura corporal, obesidade, arteriosclerose e doenças cardiovasculares, tendo como agravante o sedentarismo ${ }^{3}$. Isso não significa que todos os idosos se tornarão dependentes de outras pessoas, embora haja indicação de que $25 \%$ dessa população chegará ao estado de dependência para realizar as atividades do cotidiano ${ }^{4}$.

A atividade física pode reduzir o risco de diferentes doenças e a sua promoção tem sido objeto de campanhas públicas em diversos países ${ }^{5}$. É definida como gasto energético por meio de movimentos corporais produzidos pela musculatura esquelética ${ }^{6}$. Contempla atividades em diversos contextos como lazer, meio de transporte, tarefas domésticas e emprego pago. Fatores ambientais, intra e interpessoais estão associados à prática da atividade física ${ }^{7}$.

Dentre as atividades importantes para a manutenção do equilíbrio, agilidade e capacidade funcional, as mais utilizadas são as caminhadas, exercícios de força e alongamento. Os exercícios físicos regulares podem prevenir obesidade, diabetes, dislipidemias e doenças cardíacas. Cabe lembrar que a prática dessas atividades diminui com o avanço da idade ${ }^{1}$, o que pode se associar à diminuição da capacidade física ${ }^{8}$. Sabe-se também que mulheres têm menor atividade física em relação aos homens, principalmente em atividades de lazer; porém, participam mais do que os homens em atividades caseiras 9 . A Associação Médica Brasileira (AMB), o Conselho Federal de Medicina (CFM) e a Sociedade Brasileira de Medicina do Esporte (SBME) recomendam aos profissionais da saúde que estimulem a prática de atividades físicas regulares com o objetivo de diminuir o risco de doenças ${ }^{10}$.

Estudo que investigou os efeitos do nível de atividade física e morbi/mortalidade feminina concluiu que mulheres que realizavam atividades recreativas enquanto jovens e durante a transição menopausal mantiveram um alto nível de atividade física posteriormente, e menor morbi/mortalidade ${ }^{11}$.

Em pesquisa realizada nas capitais brasileiras, na qual se considerou a inatividade como ausência de qualquer de atividade, 53,2\% das mulheres com mais de 65 anos referiram serem inativas ${ }^{12}$. Outros estudos consideram o ponto de corte de 150 minutos/semana de prática para a definição dos níveis de atividade física ${ }^{13,14}$. Em um grupo de 262 idosos, 225 do sexo feminino e 37 do sexo masculino, com média de idade de 70,5 anos, verificou-se que 88,7\% foram considerados ativos (praticavam $\geq 150$ minutos/ semana) e $11,3 \%$ insuficientemente ativos (praticavam $<150$ minutos/semana) ${ }^{14}$. Usando o mesmo critério, uma investigação abordando o nível de atividade física, condições de saúde e características sociodemográficas de mulheres idosas brasileiras mostrou que um terço destas eram pouco ativas, e dois terços, ativas ${ }^{13}$. Pesquisa no Estado de São Paulo que levou em conta, além dos 150 minutos semanais, a intensidade dos exercícios, constatou que, das mulheres com idade entre 50 e 59 anos, $1,5 \%$ eram muito ativas; $52,5 \%$, ativas; $40,6 \%$, irregularmente ativas; e $5,4 \%$, sedentárias. Entre as mulheres com mais de 70 anos, observou-se que 9,6\% eram sedentárias ${ }^{15}$. Quando foram avaliados os diferentes domínios da atividade física, estudo realizado com mulheres na pós-menopausa mostrou que $83,5 \%$ eram ativas, sendo que as principais atividades eram os trabalhos domésticos e a caminhada como forma de locomoção ${ }^{16}$.

A atividade física, além dos benefícios físicos, parece ter um papel importante na qualidade de vida, que engloba domínios como capacidade funcional, estado emocional, interação social, atividade intelectual, autocuidado, suporte familiar, estado de saúde, valores culturais, éticos, religiosos, estilo de vida, satisfação com o emprego e/ou com atividades diárias e ambiente em que se vive. Está relacionada à autoestima e ao bem-estar individual ${ }^{17}$.

Em estudos sobre qualidade de vida em idosos, Vecchia et al. ${ }^{17}$ relataram que $36,2 \%$ interpretaram qualidade de vida como valorizar os relacionamentos interpessoais, equilíbrio emocional e boa saúde; 39,7\% valorizaram hábitos saudáveis, lazer e bens materiais; enquanto $24,1 \%$ valorizaram espiritualidade, trabalho, retidão e caridade, conhecimento e ambientes favoráveis. 
Xavier et al. ${ }^{18}$ relataram que, para alguns, envelhecer é uma etapa de desenvolvimento e satisfação e que, para outros, é um momento negativo da vida.

Em estudo de revisão realizado recentemente nos Estados Unidos abordando atividade física entre os adultos idosos, constatou-se que há uma taxa alarmante de inatividade física; existem fortes evidências dos efeitos benéficos da atividade física nas deficiências funcionais e em aspectos relacionados à qualidade de vida dessa população; há uma sustentação emergente da importância da autonomia para a prática de atividade física e os resultados na qualidade de vida dos adultos idosos ${ }^{19}$.

No entanto, há ainda poucos estudos na literatura médica, especialmente na nacional, sobre a associação entre o nível de atividade física e a qualidade de vida em mulheres com mais de 60 anos. As pesquisas disponíveis geralmente só contemplam a avaliação do índice da atividade física associado às características sociodemográficas da população. Considerando que a prática regular de atividades físicas pode diminuir as comorbidades mais prevalentes nessa faixa etária, assim como aumentar a sensação de bem-estar, este estudo teve como objetivo conhecer o nível de atividade física, a qualidade de vida, os fatores associados e a relação entre atividade física e qualidade de vida em mulheres com 60 anos ou mais. Com esses dados, espera-se contribuir para que os profissionais da saúde ofereçam uma orientação mais adequada a essas mulheres, para que possam viver mais e melhor.

\section{Métodos}

Estudo de corte transversal que incluiu 271 mulheres com idade de 60 anos ou mais, frequentadoras de qualquer um dos diversos tipos de atividade (costura, dança, leitura, pintura, ginástica entre outros) oferecidos pelo Serviço Social do Comércio de Campinas (SESC/Campinas), e mulheres em acompanhamento no Ambulatório de Menopausa, no Hospital da Mulher Professor José Aristodemo Pinotti e no Centro de Atenção Integral à Saúde da Mulher (CAISM) da Universidade Estadual de Campinas (UNICAMP) no período entre junho de 2009 e junho de 2010 . O protocolo desta pesquisa foi aprovado pelo Comitê de Ética em Pesquisa da Faculdade de Ciências Médicas da UNICAMP, e as participantes assinaram termo de consentimento livre e esclarecido (TCLE). Foram excluídas mulheres com dificuldade de responder os questionários ou com comprometimento físico severo que impossibilitasse a prática de qualquer atividade física.

O tamanho amostral foi calculado assumindo-se um nível de significância de 5\% (poder do teste de $80 \%$ ), segundo o teste $t$, com uma diferença de no mínimo $10 \%$ entre as médias das seções do questionário de atividade física. Verificou-se serem necessários 57 sujeitos para cada nível de atividade física. Considerando a prevalência de idosos pouco ativos de $21 \%$, e com atividade moderada/ ativa de $79 \%^{20}$, foram avaliadas 271 mulheres.

O processo de seleção foi ao acaso em ambos os ambientes de pesquisa. No SESC/Campinas, as mulheres foram abordadas nos espaços da instituição e convidadas a participar. No Ambulatório de Menopausa do CAISM, as mulheres foram selecionadas pelo prontuário de atendimento para verificação da elegibilidade antes da consulta médica. Depois de selecionadas, as mulheres leram e assinaram o TCLE. Não houve recusas para participar da pesquisa. As mulheres foram submetidas à entrevista, conduzida pelo pesquisador principal. Foram coletadas informações referentes a características como idade, peso, altura, estado marital, escolaridade, tabagismo, autopercepção do estado de saúde, número de medicamentos em uso e antecedentes de doenças. A classificação econômica foi realizada de acordo com as recomendações da Associação Brasileira de Empresas de Pesquisa.

O nível de atividade física foi avaliado pelo Questionário Internacional de Atividade Física - Versão 8, forma longa, (IPAQ-Versão 8.0), validado no Brasil em 2004 por Benedetti et al. ${ }^{21}$ e utilizado em pesquisas anteriores com mulheres na pós-menopausa ${ }^{8}$. É um instrumento que permite estimar o tempo semanal gasto na realização de atividades físicas de acordo com a intensidade em diferentes contextos da vida (trabalho, tarefas doméstica, transporte e lazer). Há a possibilidade de estimar o tempo despendido em atividades menos intensas, como as realizadas na posição sentada. Esse questionário considera como atividade física todo movimento corporal que envolva qualquer esforço físico. Esses esforços são divididos em vigorosos (os que precisam de um grande esforço físico) e moderados (aqueles que exigem algum esforço físico). O questionário consta de cinco seções. A seção 1 inclui perguntas sobre a presença de atividades físicas no trabalho voluntário ou remunerado fora de casa. A seção 2 consta de perguntas sobre atividade física como meio de transporte (caminhadas ou uso de automóvel ou bicicleta). A seção 3 tem questões sobre atividade física em casa, tarefas domésticas e atenção à família. A seção 4 inclui questionamentos sobre a prática de atividade física de recreação, esporte, exercício e lazer. Por fim, a seção 5 aborda as atividades realizadas quando a mulher se encontra sentada. No caso das mulheres que praticaram cada uma das atividades explicitadas nas cinco seções, perguntouse sobre a duração e a frequência dessas atividades. A pontuação final foi expressa pela somatória do tempo de atividade física, em minutos, na semana anterior ao estudo, em cada seção do questionário. A classificação do IPAQ foi realizada segundo critérios utilizados em estudos prévios em que, para a classificação em três níveis de atividade física, foram ordenados, de modo crescente, os valores 
totais e, em seguida, realizados dois cortes aos $33 \%$ e $66 \%$, classificando as mulheres em pouco, moderadas e muito ativas (técnica conhecida como tercis) ${ }^{21}$.

A qualidade de vida foi avaliada pelo questionário da Organização Mundial da Saúde, WHOQOL-OLD, específico para idosos, validado no Brasil por Fleck et al. ${ }^{22}$ em 2006. O questionário é dividido em seis domínios. $\mathrm{O}$ primeiro refere-se às habilidades sensoriais e envolve perguntas que relacionam os sentimentos com os cinco sentidos (audição, visão, paladar, olfato e tato). O segundo domínio, a autonomia, aborda os sentimentos relacionados a decisões e à capacidade de realizar atividades. $\mathrm{O}$ terceiro domínio questiona sobre atividades e sentimentos atuais, passados e futuros. O quarto avalia a participação social, o envolvimento dos sentimentos e a integração social. $\mathrm{O}$ quinto domínio, relacionado à morte e ao morrer, questiona as preocupações e o medo dessa situação. O sexto domínio, intimidade, tem perguntas sobre os sentimentos de amor e companheirismo. Quanto maior a pontuação (escore), melhor a qualidade de vida, sendo que zero corresponde à pior e cem à melhor qualidade de vida.

Os dados foram analisados por meio de média, desvio padrão, mediana e frequência. Para cada sujeito foi aferido o quanto cada seção do questionário contribuiu para o tempo total de atividades (IPAQ) e calculado o percentual médio para cada item. O tempo total de atividades (IPAQ) foi avaliado através de tercis (percentis 33,3\% e $66,6 \%$ ) para classificar a amostra quanto ao IPAQ como pouco ativa e ativa ${ }^{21}$. O teste do qui-quadrado avaliou as associações entre o IPAQ e as características das mulheres. As associações entre os domínios da qualidade de vida, as características das mulheres e o IPAQ foram através do teste $t$ (ou Mann-Whitney). As análises multivariadas foram realizadas por meio de regressão logística e regressão linear com critério de seleção de variáveis stepwise. O nível de significância foi de $5 \%$, e o software utilizado para análise foi o SAS versão 9.1.3.

\section{Resultados}

Foram entrevistadas 271 mulheres, sendo 130 (48\%) frequentadoras das atividades de um centro de lazer, o Serviço Social do Comércio em Campinas (SP), e 141 (52\%) do Ambulatório de Menopausa do CAISM/ UNICAMP. A média de idade foi de 67,4 ( \pm 5,3 anos), sendo que $70,1 \%$ dessas mulheres apresentavam idade entre 60 a 69 anos. Na classificação de índice de massa corpórea (IMC), a maioria foi classificada portadora de sobrepeso (38\%) e obesa $(33,2 \%)$. Da amostra estudada, $50,2 \%$ viviam com companheiro e 40,6\% referiram nível de escolaridade entre o primeiro grau completo e segundo grau incompleto. Quando interrogadas sobre tabagismo, a maioria $(73,8 \%)$ afirmou que não fumava. Questionadas sobre a autopercepção da saúde, 59,7\% reportaram terem saúde excelente, muito boa ou boa. A maioria das mulheres $(70,8 \%)$ fazia uso de um a quatro medicamentos, e (90\%) apresentaram histórico de algum tipo de doença pregressa. Em relação à classe social, 47,2\% das mulheres pertenciam à classe $\mathrm{C}$ (tabelas não-apresentadas).

A análise dos dados da seção 1 do questionário de atividade física (IPAQ) mostrou que 32,5\% das mulheres reportaram realizar trabalho remunerado ou voluntário fora de casa. Dessas, 1,8\% referiram realizar atividades no trabalho de intensidade vigorosa, e 9,6\% de atividade moderada. Daquelas que realizaram algum tipo de trabalho, $16,6 \%$ faziam caminhada no emprego. Na seção 2 (atividades como meio de transporte), as entrevistadas disseram utilizar automóvel $(87,5 \%)$, caminhadas de um lugar para o outro $(77,1 \%)$ e bicicleta $(1,8 \%)$ para se deslocarem. $\mathrm{Na}$ análise da seção 3 (atividades domésticas), verificou-se que, respectivamente, $4,8 \%$ e $38 \%$ das mulheres realizaram atividades vigorosas e moderadas no jardim ou quintal, e 83,4\% realizaram atividades moderadas em casa na semana anterior à entrevista. Com relação aos resultados da seção 4, 43,2\% das mulheres fizeram caminhadas como exercício, 13,3\% realizaram exercícios físicos vigorosos e $50,6 \%$ das relataram fazer exercícios físicos moderados (tabelas não-apresentadas).

Observou-se que o tempo médio de atividade física foi de $2.802,7 \pm 1.154,9$ minutos na semana anterior à entrevista, ou seja, 46,6 horas em 7 dias. A análise de cada pergunta das seções do índice de atividade física mostrou que $60,8 \%$ dessas horas foi o tempo em atividade física sentada com média de 1.701,6士986,1 minutos na semana, e $13,0 \%$ em atividades moderadas em casa. As demais atividades foram de pouca representatividade no índice de atividade física total (Tabela 1). Não houve associação significativa entre as características das mulheres e o índice de atividade física (IPAQ) total.

Da amostra completa de mulheres, $63,9 \%$ realizavam exercícios físicos moderados ou vigorosos. Associando as características das mulheres com a prática de exercícios físicos de intensidade moderada ou vigorosa, verificou-se, por meio da análise múltipla, que as variáveis significativamente associadas à prática de exercícios físicos moderados/vigorosos foram a origem e a idade. As mulheres frequentadoras do centro de lazer apresentaram 11,4 mais chances de praticarem exercícios físicos moderados/ vigorosos, e as com idade acima de 70 anos apresentaram chance 2,8 vezes maior (Tabela 2 ).

A Tabela 3 mostra que o escore médio de qualidade de vida total foi de $66,9 \pm 11,7$. O maior valor foi observado no domínio referente às habilidades sensoriais $(72,0 \pm 18,8)$, e o menor, no que se refere à autonomia $(60,3 \pm 16,2)$.

Avaliando as características das mulheres e a prática de exercícios físicos com o escore de qualidade de vida 
por meio da regressão linear múltipla, evidenciou-se que

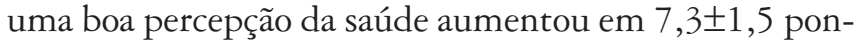
tos o escore de qualidade de vida. Quanto maior o uso de medicamentos, menor a pontuação no escore de qualidade de vida (um a quatro diminuiu 2,2 $\pm 1,4$ pontos; e cinco ou mais diminuiu 4,4 $\pm 1,4$ pontos). Praticar exercícios físicos moderado/vigorosos aumentou em $4,8 \pm 1,5$ pontos o escore de qualidade de vida (dados não-apresentados).

Tabela 1 - Índice de Atividade Física (IPAQ Total) e percentual de representatividade em cada seção

\begin{tabular}{|c|c|c|c|c|}
\hline \multirow{2}{*}{ Variável } & \multirow{2}{*}{$n$} & \multirow{2}{*}{ Ordem de representatividade } & \multicolumn{2}{|c|}{$\%$ médio de representatividade no IPAQ } \\
\hline & & & Média & Desvio padrão \\
\hline \multicolumn{5}{|l|}{ IPAQ } \\
\hline Trabalho & 54 & & 3,8 & 11,1 \\
\hline Atividades vigorosas no trabalho & 5 & $\left(12^{\circ}\right)$ & 0,1 & 1,7 \\
\hline Atividades moderadas no trabalho & 26 & $\left(9^{\circ}\right)$ & 1,3 & 5,8 \\
\hline Caminhada no trabalho & 45 & $\left(8^{\circ}\right)$ & 2,3 & 8,1 \\
\hline Andar de bicicleta & 5 & $\left(13^{\circ}\right)$ & 0,1 & 1,1 \\
\hline Caminha de um lugar para outro & 209 & $\left(4^{\circ}\right)$ & 3,9 & 4,3 \\
\hline Tarefas domésticas & 232 & & 15,9 & 14,0 \\
\hline Atividades vigorosas em casa & 13 & $\left(11^{\circ}\right)$ & 0,5 & 3,8 \\
\hline Atividades moderadas no jardim ou quintal & 103 & $\left(7^{\circ}\right)$ & 2,4 & 4,9 \\
\hline Exercícios físicos vigorosos & 36 & $\left(10^{\circ}\right)$ & 0,9 & 2,9 \\
\hline Exercícios físicos moderados & 137 & $\left(5^{\circ}\right)$ & 3,8 & 5,3 \\
\hline Tempo sentada & 271 & $\left(1^{\circ}\right)$ & 60,8 & 20,4 \\
\hline Total & 271 & & & \\
\hline
\end{tabular}

Tempo total médio de atividade física (IPAQ) 2.802,7 minutos/semana.Ordem de representatividade: percentual de tempo na atividade em relação ao tempo total.

Tabela 2 - Características das mulheres associadas à prática de exercícios físicos de intensidade moderado/vigorosa

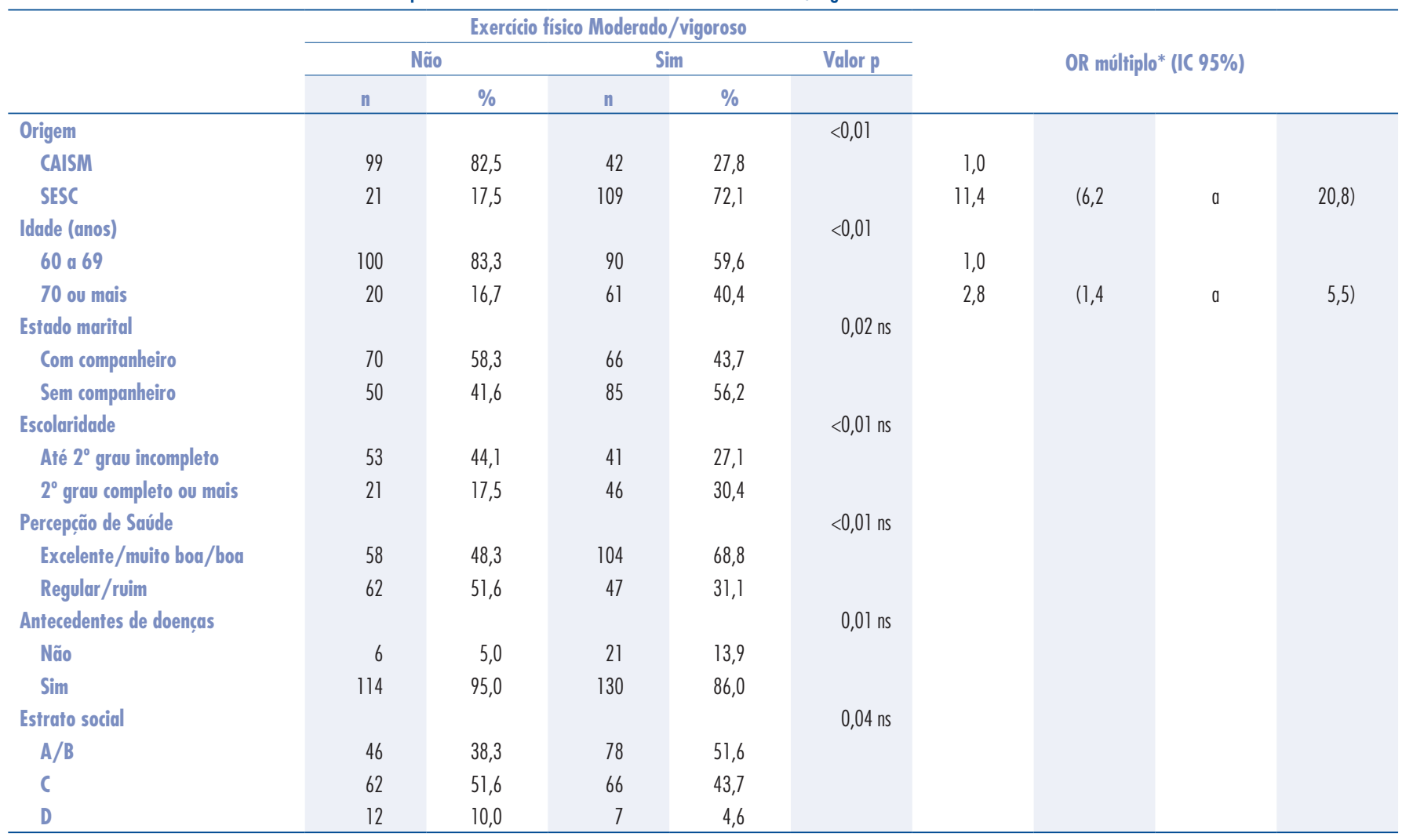

ns=não significativo / IC=Intervalo de Confiança / OR=Odds Ratio. teste de qui-quadrado / *regressão logística múltipla com critério de seleção de variáveis stepwise. 
Quando se avaliou o índice de atividade física total e o escore de qualidade de vida em cada domínio não se evidenciou associação significativa. Porém, quando se testou essa associação de acordo com a intensidade da atividade física, verificou-se que quem referiu a prática de exercícios físicos moderado/vigorosos apresentou maiores escores de qualidade de vida em praticamente todos os domínios. A exceção foi o domínio relacionado à morte e morrer (Tabela 4).

Tabela 3 - Qualidade de Vida (WHOQOL-OLD) e distribuição da pontuaç̃õo em cada domínio

\begin{tabular}{l|c|c|c}
\hline Variável & n & Média & Desvio padrão \\
\hline WHOQOL-OLD & & & \\
$\quad$ Habilidade sensorial & 271 & 72,0 & 18,8 \\
\hline Atividades passadas, presentes e fułuras & 271 & 68,5 & 16,6 \\
\hline Intimidade & 271 & 68,1 & 18,0 \\
\hline Participação social & 271 & 67,6 & 17,1 \\
Morłe e morrer & 271 & 64,9 & 23,2 \\
Autonomia & 271 & 60,3 & 16,2 \\
Total & 271 & 66,9 & 11,7 \\
\hline
\end{tabular}

0 (zero): pior escore; e 100 (cem): melhor escore de qualidade de vida.

Tabela 4 - Qualidade de vida e atividade física

\begin{tabular}{|c|c|c|c|c|}
\hline & \multicolumn{2}{|c|}{ IPAQ Total } & \multicolumn{2}{|c|}{$\begin{array}{c}\text { Exercícios Físicos } \\
\text { Moderados/Vigorosos }\end{array}$} \\
\hline & Pouco & Ativa & Não & Sim \\
\hline \multicolumn{5}{|l|}{ Habilidade sensorial } \\
\hline Média & 70,5 & 74,2 & 67,7 & 77,1 \\
\hline Desvio-padrão & 19,7 & 18,3 & 19,8 & 16,9 \\
\hline Valor $\mathbf{p}$ & \multicolumn{2}{|c|}{0,16} & \multicolumn{2}{|c|}{$<0,01$} \\
\hline \multicolumn{5}{|l|}{ Autonomia } \\
\hline Média & 60,3 & 60,4 & 56,6 & 63,3 \\
\hline Desvio-padrão & 15,5 & 16,5 & 16,0 & 15,7 \\
\hline Valor $p$ & \multicolumn{2}{|c|}{0,88} & \multicolumn{2}{|c|}{$<0,01$} \\
\hline \multicolumn{5}{|l|}{$\begin{array}{l}\text { Atividades passadas } \\
\text { presentes e futuras }\end{array}$} \\
\hline Média & 69,1 & 68,2 & 64,3 & 71,9 \\
\hline Desvio-padrão & 15,4 & 17,3 & 17,4 & 15,2 \\
\hline Valor $p$ & \multicolumn{2}{|c|}{0,95} & \multicolumn{2}{|c|}{$<0,01$} \\
\hline \multicolumn{5}{|l|}{ Participação social } \\
\hline Média & 69,2 & 66,8 & 62,3 & 71,8 \\
\hline Desvio- padrão & 15,6 & 17,8 & 17,4 & 15,1 \\
\hline Valor p & \multicolumn{2}{|c|}{0,36} & \multicolumn{2}{|c|}{$<0,01$} \\
\hline \multicolumn{5}{|l|}{ Morte e morrer } \\
\hline Média & 61,7 & 66,5 & 64,4 & 65,2 \\
\hline Desvio padrão & 23,3 & 23,1 & 22,5 & 23,8 \\
\hline Valor $p$ & \multicolumn{2}{|c|}{0,10} & \multicolumn{2}{|c|}{0,59} \\
\hline \multicolumn{5}{|l|}{ Intimidade } \\
\hline Média & 68,8 & 67,8 & 65,3 & 70,3 \\
\hline Desvio padrão & 17,7 & 18,1 & 18,8 & 17,0 \\
\hline Valor $p$ & \multicolumn{2}{|c|}{0,76} & \multicolumn{2}{|c|}{0,02} \\
\hline
\end{tabular}

Utilizado teste de Mann-Whitney.

\section{Discussão}

O presente estudo teve como objetivo avaliar o nível de atividade física, a qualidade de vida e os fatores associados em mulheres frequentadoras de um centro de lazer e do Ambulatório de Menopausa. Cerca de um terço das mulheres foram categorizadas como pouco ativas. Mais relevante que isso, constatou-se que mulheres com 60 anos ou mais realizam predominantemente atividades físicas na posição sentada, e poucas atividades de intensidade moderada ou vigorosa no trabalho, transporte e lazer. Esses resultados são consistentes com outros estudos que avaliaram tanto idosas brasileiras ${ }^{13}$ quanto não-brasileiras ${ }^{10,23}$, e também constataram que essas mulheres despendem muito tempo na posição sentada para assistir televisão, locomoverem-se nos automóveis, ouvir rádio, costurar, ler e receber visitas. O estudo de Pate et al. mostrou que idosas menos ativas ficavam de 10 a 15 horas sentadas, enquanto as mais ativas, entre 5 e 9 horas $^{24}$. Considerando as recomendações atuais de se praticar pelo menos 30 minutos diários de atividade física com intensidade moderada ou vigorosa para se conseguir benefícios em relação à saúde ${ }^{13,24}$, essa população está muito aquém de obter os benefícios que podem advir com a prática de atividades físicas regulares, pois atividades realizadas na posição sentada são consideradas de baixo gasto energético e associam-se ao sedentarismo, ao risco de obesidade e às suas consequências, como as doenças cardiovasculares e dependência física ${ }^{25,26}$.

$\mathrm{Na}$ análise múltipla, frequentar um centro de lazer e ter maior idade foram as principais características associadas à prática de exercícios moderados ou intensos. Estudo feito em outro contexto confirmou a importância de se ter acesso e frequentar um centro de lazer, uma vez que fatores ambientais, intra e interpessoais estão associados à realização da atividade física ${ }^{7}$.

Os dados da literatura são conflitantes em relação às características das mulheres e à intensidade da atividade física. Alguns estudos verificaram que ser mulher com idade acima de 80 anos, viúva, sem escolaridade e não realizar trabalhos voluntários foram variáveis associadas ao sedentarismo ${ }^{13,15}$. Mulheres com maior escolaridade, maior renda e família menor reportam não se exercitarem, enquanto a presença de doenças crônicas associa-se positivamente à prática de exercícios esportivos. O fato é que a comparação de resultados é uma tarefa difícil, uma vez que avaliouse uma população demasiadamente específica, ou seja, frequentadoras de um centro de lazer e cultura ou de um serviço de saúde terciário, localizados em Campinas (SP). Diante disso, exigiu-se cautela nas 
comparações com estudos de outros pesquisadores nacionais ou estrangeiros.

O escore médio de qualidade de vida foi $66,9 \pm 11,7$, para um máximo de 100. Apesar de o estudo não ter utilizado grupos de comparação, pode-se inferir que a qualidade de vida dessas mulheres foi relativamente boa, pois está acima da média, considerando o valor máximo que poderia ser observado. Cabe lembrar que a qualidade de vida depende das expectativas individuais e do meio no qual o indivíduo está inserido. $\mathrm{O}$ maior valor observado foi no domínio habilidade sensorial, e o pior, na autonomia. Quanto às habilidades sensoriais, o resultado pode ser considerado dentro do esperado pelo fato de essas mulheres provavelmente apresentarem capacidades de interação com o ambiente e outras pessoas, uma vez que são frequentadoras de instituições geralmente distantes de suas moradias.

O domínio autonomia teve o menor escore, o que pode indicar que essas mulheres apresentaram certo grau de dependência de outras pessoas em suas atividades diárias. Frequentadoras de um centro de lazer, sem companheiro, analfabetas ou com primeiro grau incompleto, com boa autopercepção de saúde, que não relataram uso de medicamentos, sem doenças, com maior renda e praticantes de exercícios físicos de intensidade moderada ou vigorosa apresentaram escores de qualidade de vida significativamente maiores. Na regressão linear evidenciou-se que ter uma boa saúde aumentou 7,3 pontos o escore de qualidade de vida; referir o uso de maior número de medicamentos diminuiu o escore em 4,4 pontos; e quem praticou exercícios físicos de intensidade moderada ou vigorosa aumentou em 4,8 pontos o referido escore. Sprangers et al.$^{26}$ reportaram dados discordantes. Descreveram que a ausência de companheiro, idade avançada e baixo nível de escolaridade foram associados a menores escores de qualidade de vida. Esses pesquisadores avaliaram a qualidade de vida em indivíduos de ambos os sexos de diversas faixas etárias portadores de doenças crônicas.

Exercícios físicos de intensidade moderada/vigorosa se associaram significativamente a maiores escores do WHOQOL-OLD em quase todos os domínios. Estudos recentes também ressaltam o papel do domínio autonomia ${ }^{27}$, assim como a participação dos idosos em centros de lazer, para uma melhor qualidade de vida ${ }^{5}$. O único domínio que não se associou à qualidade de vida foi o relacionado à morte e ao morrer. Esse resultado pode ser considerado esperado, pois as pessoas em geral, e especialmente as de maior idade, têm consciência da finitude da vida, e isso nem sempre está relacionado a determinadas atitudes ou práticas.

Considerando que a recomendação para uma melhora nas condições físicas, na manutenção da saúde e, sobretudo, na qualidade de vida, é a prática de atividades físicas de intensidade moderada ou vigorosa por 150 minutos ou mais por semana ${ }^{9,23,28-30}$, a população avaliada neste estudo está aquém desse ideal. O fato que não deve ser ignorado é o crescimento expressivo da população idosa com pouca prática de atividades físicas moderadas e vigorosas, o que pode acarretar decréscimo precoce da capacidade física. O quadro é preocupante, pois sabe-se que além de haver uma prevalência alta de sedentarismo nessa população, existe uma prevalência ainda maior de pessoas que não atingem as recomendações mínimas de atividade física para a manutenção da saúde ${ }^{15}$. Sabe-se também da relação linear entre o nível de atividade física e o retardo do declínio das funções do organismo, resultando na melhoria do estado de saúde do indivíduo ${ }^{31}$, e, com isso, podendo melhorar a qualidade de vida ${ }^{17}$.

Finalmente destaca-se que os resultados deste estudo não devem ser generalizados a todas as idosas brasileiras, pois avaliamos mulheres frequentadoras de um serviço de saúde de referência terciária especializado no atendimento de mulheres na pós-menopausa, ou usuárias das atividades disponíveis em um centro de lazer da cidade de Campinas (SP), que também atende uma população com características e necessidades específicas. Essas características e necessidades as diferem de outras populações. Além disso, a natureza do estudo só permitiu estabelecer associações entre as variáveis, e não relações de causa-efeito. Por esses fatos, novas pesquisas são necessárias, prospectivas, idealmente de base populacional, com avaliações antes e depois de determinadas intervenções, e, preferencialmente, com grupos para comparação. Vale ressaltar, por outro lado, a seleção ao acaso dos sujeitos, único entrevistador e a utilização de questionários validados nacionalmente, adaptados para essa população. Assim, acredita-se que os resultados podem auxiliar as mulheres e os profissionais que as assistem, pois ficou claro que são necessárias ações que incrementem o nível de atividade física dessa população. Se houver conscientização e ações específicas nessa direção, pode-se sonhar com um futuro de envelhecimento melhor, com autonomia e independência para todos. 
1. Franchi KMB, Montenegro RM. Atividade física: uma necessidade para a boa saúde na terceira idade. Rev Bras Promoção Saúde. 2005; 18(3):152-6.

2. Guimarães LHCT, Galdino DCA, Martins FLM, Vitorino DFM, Pereira KL, Carvalho EM. Comparação da propensão de quedas entre idosos que praticam atividade física e idosos sedentários. Rev Neurociências. 2004;12(2):68-72.

3. Martins RA, Veríssimo MT, Coelho e Silva M, Cumming SP, Teixeira AM. Effects of aerobic and strength-based training on metabolic health indicators in older adults. Lipidis Health Dis. 2010;9:76.

4. Andreotti RA, Okuma SS. Validação de uma bateria de testes de atividades da vida diária para idosos fisicamente independentes. Rev Paul Educ Fís. 1999;13(1):46-66.

5. Juraki D, Pediši Z, Greblo Z. Physical activity in different domains and health-related quality of life: a population-based study. Qual Life Res. In Press 2010.

6. Sims J, Hill K, Hunt S, Haralambous B. Physical activity recommendations for older Australians. Australas J Ageing. 2009;29(2):81-7.

7. Annear M, Cushman G, Gidlow B. Leisure time physical activity differences among older adults from diverse socioeconomic neighborhoods. Health Place. 2009;15(2):482-90.

8. Ferreira M, Matsudo S, Matsudo V, Braggion G. Efeitos de um programa de orientação de atividade física e nutricional sobre o nível de atividade física de mulheres fisicamente ativas de 50 a 72 anos de idade. Rev Bras Med Esporte. 2005; 11 (3):172-6.

9. Jurj AL, Wen W, Gao YT, Matthews CE, Yang G, Li HL, et al. Patterns and correlates of physical activity: a cross-sectional study in urban Chinese women. BMC Public Health. 2007;7:213.

10. Silva RB, Costa-Paiva L, Pinto Neto AM, Braga AA, Moraes SS. Atividade física habitual e risco cardiovascular na pós-menopausa. Rev Assoc Med Bras. 2006;52(4):242-6

11. Nitz JC, Choy NL. Changes in activity level in women aged $40-80$ years. Climacteric. 2007;10(5):408-15.

12. Brasil. Ministério da Saúde [Internet]. Vigitel Brasil 2009: vigilância de fatores de risco e proteção para doenças crônicas por inquérito telefônico. Brasília (DF): Ministério da Saúde; 2010 [citado 2010 Jul 16]. Disponível em: <http://portal.saude.gov.br/portal/ arquivos/pdf/vigitel_2009_preliminar_web.pdf>

13. Mazo GZ, Mota J, Gonçalves LHT, Matos MG. Nível de atividade física, condições de saúde e características sócio-demográficas de mulheres idosas brasileiras. Rev Port Cien Desp. 2005;5(2):202-12.

14. Cardoso AS, Levandoski G, Mazo GZ, Prado APM, Cardoso LS. Comparação do nível de atividade física em relação ao gênero de idosos participantes de grupos de convivência. Rev Bras Ciênc Envelhec Hum. 2008;5(1):9-18.

15. Matsudo SM, Matsudo VR, Araúio T, Andrade D, Andrade E, Oliveira LC, et al. Nível de atividade física da população do Estado de São Paulo: análise de acordo com o gênero, idade, nível socioeconômico, distribuição geográfica e de conhecimento. Rev Bras Cien e Mov. 2002;10(4):41-50.

16. Da Silva RB, Costa-Paiva L, Pinto-Neto AM, Braga Ade A, Morais SS. Association between habitual physical activity and parameters of physical fitness in postmenopausal women. Climacteric. 2005;8(4):360-70.

17. Vecchia RD, Ruiz T, Bocchi SCM, Corrente JE. Qualidade de vida na terceira idade: um conceito subjetivo. Rev Bras Epidemiol. 2005;8(3):246-52.

18. Xavier FM, Ferraz MP, Marc N, Escosteguy NU, Moriguchi EH. Elderly people's definition of quality of life. Rev Bras Psiquiatr. 2003;25(1):31-9.

19. Motl RW, McAuley E. Physical activity, disability, and quality of life in older adults. Phys Med Rehabil Clin N Am. 2010;21(2): 299-308.

20. Mazo GZ, Liposcki DB, Ananda C, Preve D. Condições de saúde, incidência de quedas e nível de atividade física dos idosos. Rev Bras Fisioter. 2007; 11 (6):437-42.

21. Benedetti TB, Mazo GZ, Barros MVG. Aplicação do questionário internacional de atividades físicas para avaliação do nível de atividades físicas de mulheres idosas: validade concorrente e reprodutibilidade teste-reteste. Rev Bras Cien Mov. 2004;12(1): 25-34.

22. Fleck MP, Chachamovich $E$, Trentini $C$. Development and validation of the Portuguese version of the WHOQOL-OLD module. Rev Saude Publica. 2006;40(5):785-91.

23. Gierach GL, Chang SC, Brinton LA, Lacey JV Jr, Hollenbeck AR, Schatzkin A, et al. Physical activity, sedentary behavior, and endometrial cancer risk in the NHI-AARP Diet and Health Study. Int J Cancer. 2009; 124(9):2 139-47.

24. Pate RR, Pratt M, Blair SN, Haskell WL, Macera CA, Bouchard C, et al. Physical activity and public health. A recommendation from the Centers for Disease Control and Prevention and the American College of Sports Medicine. JAMA. 1995;273(5):402-7.

25. Stewart KJ. Physical activity and aging. Ann N Y Acad Sci. 2005; 1055:193-206.

26. Dunn MZ. Psychosocial mediators of a walking intervention among African American women. J Transcult Nurs. $2008 ; 19(1): 40-6$.

27. Sprangers MA, de Regt EB, Andries F, van Agt HM, Bijl RV de Boer JB, et al. Which chronic conditions are associated with better or poorer quality of life? J Clin Epidemiol. 2000;53(9):895-907.

28. White SM, Wójcicki TR, McAuley E. Physical activity and quality of life in community dwelling older adults. Health Qual Life Outcomes. 2009;7:10.

29. Lees E, Taylor WC, Hepworth JT, Feliz K, Cassells A, Tobin JN. Environmental changes to increase physical activity: perceptions of older urban ethnic-minority women. J Aging Phys Act. 2007; 15(4): 425-38.

30. Lim KC, Kayser-Jones JS, Waters C, Yoo G. Aging, health and physical activity in Korean Americans. Geriatr Nurs. 2007;28(2): 11 2-9.

31. Findorff M, Wyman JF, Gross CR. Predictors of long-term exercise adherence in a community-based sample of older women. J Womens Health (Larchmt). 2009;18(11):1769-76. 